\title{
A PROOF OF WAHL'S CONJECTURE IN THE SYMPLECTIC CASE
}

\author{
JESPER FUNCH THOMSEN
}

\begin{abstract}
Let $X$ denote a flag variety of type $A$ or type $C$. We construct a canonical Frobenius splitting of $X \times X$ which vanishes with maximal multiplicty along the diagonal. This way we verify a conjecture by Lakshmibai, Mehta and Parameswaran 4 in type $C$, and obtain a new proof in type $A$. In particular, we obtain a proof of Wahl's conjecture in type $C$, and a new proof in type $A$. We also present certain cohomological consequences.
\end{abstract}

\section{INTRODUCTION}

Let $X=G / P$ denote a generalized flag variety over an algebraically closed field $k$. Let $\mathcal{J}$ denote the sheaf of ideals defining the diagonal subvariety $\Delta(X)$ of $X \times X$. The sheaf of differential 1-forms $\Omega_{X}$ on $X$ then equals the quotient $\mathrm{J} / \mathrm{J}^{2}$ and consequently we have a surjective map

$$
\mathrm{J} \rightarrow \Delta_{*}\left(\Omega_{X}\right),
$$

of sheaves on $X \times X$. Whenever $\mathcal{L}_{1}$ and $\mathcal{L}_{2}$ denote locally free sheaves of rank 1 on $X$ we this way obtain an induced map

$$
\mathrm{H}^{0}\left(X \times X, \mathcal{J} \otimes\left(\mathcal{L}_{1} \otimes \mathcal{L}_{2}\right)\right) \rightarrow \mathrm{H}^{0}\left(X, \Omega_{X} \otimes \mathcal{L}_{1} \otimes \mathcal{L}_{2}\right) .
$$

In [9] J. Wahl conjectured that the map (11) is surjective whenever $\mathcal{L}_{1}$ and $\mathcal{L}_{2}$ are ample. In characteristic zero (which is actually the setup in [9]) this conjecture has been proved by S. Kumar in [3] using a case by case approach. At the same time Kumar remarked that a unified approach would be more satisfactory and suggestion the notion of Frobenius splitting as a feasible tool. This suggestion was later studied in [4].

In [4] the characteristic $p>0$ version of Wahl's conjecture was studied. Here it was observed that Wahl's conjecture would follow if $X \times X$ admits a Frobenius splitting vanishing with maximal multiplicity along the diagonal. The latter statement was proved to be equivalent to the existence of a Frobenius splitting of the blow-up of $X \times X$ at $\Delta(X)$ which is compatible with the exceptional locus; i.e. with the projectivized tangent bundle to $X$. This statement was then conjectured (from now on called the LMP-conjecture) to be satisfied for generalized flag varieties. 
As a first approximation to the LMP-conjecture the paper [4] gave a construction of a Frobenius splitting of $X$ vanishing with maximal multiplicity along a point. The LMP-conjecture itself was only handled for $\mathrm{SL}_{n}(k) / P$ when $n \leq 6$. Subsequently the LMP-conjecture was verified for Grassmannians by Mehta and Parameswaran [7], for symplectic and orthogonal Grassmannians by Lakshmibai, Raghavan and Sankaran [5] and by Brown and Lakshmibai for minuscule $G / P$ [2]. In all these cases the proof relied on the fact that a certain "canonical" and well studied Frobenius splitting of $X \times X$ vanished with maximal multiplicty along the diagonal. At the same time it was observed that this "canonical" Frobenius splitting would not work in general. Recently Lauritzen and Thomsen [6] introduced a new Frobenius splitting of $X \times X$, for $X=$ $\mathrm{SL}_{n}(k) / B$, with the desired vanishing property along the diagonal. As a consequence the LMP-conjecture is now verified for any generalized flag variety of the form $\mathrm{SL}_{n}(k) / P$. The paper [loc.cit] also verified the natural generalization of the LMP-conjecture for arbitrary Kempf varieties in $\mathrm{SL}_{n}(k) / B$.

In the present paper we obtain the Frobenius splitting from [6] in a new way. This reveals that the Frobenius splitting of [loc.cit] is actually a canonical Frobenius splitting. Moreover, it also reveals its relation to the above mentioned Frobenius splitting of $\mathrm{SL}_{n}(k) / B$ vanishing with maximal multiplicty along the point $e B$ [4]. It turns out that this approach generalizes naturally to the case $X=\mathrm{Sp}_{2 m}(k) / P$ and thus we also obtain a proof of the LMP-conjecture in the symplectic case . The latter should be considered as the main result of this paper. Actually, to some extend our approach also generalizes to the orthogonal case, but for some mysteries reasons certain root combinatorics prevents us from concluding the LMP-conjecture in this case.

\section{NOTATION}

2.1. Varieties. We work over a fixed algebraically closed field $k$ of positive characteristic $p>0$. By a variety we mean a reduced scheme of finite type over $k$; in particular, a variety need not be irreducible. The ring of global regular functions on $X$ is denoted by $k[X]$.

2.2. Group setup. We fix an integer $n$ and let $G=\mathrm{SL}_{n}(k)$ denote the group of $n \times n$-matrices of determinant 1 . By $B$ we denote the Borel subgroup of $G$ consisting of upper triangular matrices and let $T \subset B$ denote the maximal torus of diagonal matrices. The set of upper (resp. lower) triangular unipotent matrices in $G$ is denoted by $U$ (resp. $U^{-}$). By $I_{n}$ we denote the identity matrix in $G$.

2.3. Characters and roots. The set $X^{*}(T)$ of $T$-characters is a free abelian group of rank $n-1$. If we, for $i=1, \ldots, n$, let $\epsilon_{i}$ denote the $T$-character which picks out the $i$-th diagonal element, then the set of elements $\epsilon_{1}, \ldots, \epsilon_{n-1}$ form a basis for the group $X^{*}(T)$. The set of 
roots is the set of $T$-characters $\epsilon_{i}-\epsilon_{j}$, for $i \neq j$. A root $\epsilon_{i}-\epsilon_{j}$ is said to positive if $i<j$, while a positive root is said to be simple if it equals $\epsilon_{i}-\epsilon_{i+1}$ for some $i=1, \ldots, n-1$. A $T$-character $\lambda$ is said to be dominant if

$$
\lambda=\sum_{i=1}^{n-1} \lambda_{i} \epsilon_{i}
$$

with coefficients satisfying

$$
\lambda_{1} \geq \lambda_{2} \geq \cdots \geq \lambda_{n-1} \geq 0 .
$$

The fundamental characters $\omega_{1}, \ldots, \omega_{n-1}$ are then the dominant elements

$$
\omega_{i}=\sum_{s=1}^{i} \epsilon_{s} .
$$

2.4. Induced modules. The group of $B$-characters will be denoted by $X^{*}(B)$ and will be identified with the group of $T$-characters $X^{*}(T)$. Any $B$-character $\lambda$ defines an induced $G$-representation

$$
\operatorname{Ind}_{B}^{G}(\lambda)=\left\{f: G \rightarrow k: f(g b)=\lambda(b)^{-1} f(g) \text {, for } b \in B, g \in G\right\} .
$$

The vectorspace structure of $\operatorname{Ind}_{B}^{G}(\lambda)$ is defined in the obvious way while the action of $G$ is defined through left-translation. To simplify notation we will also use the notation $\mathrm{H}^{0}(\lambda)$ for the $G$-module $\operatorname{Ind}_{B}^{G}(-\lambda)$. Then $\mathrm{H}^{0}(\lambda)$ is nonzero if and only if $\lambda$ is dominant. Moreover, in this case $\mathrm{H}^{0}(\lambda)$ contains a unique $U^{-}$-invariant line which is called the lowest weight space of $\mathrm{H}^{0}(\lambda)$. The $T$-character associated to the lowest weight space equals $-\lambda$. The $B$-representation associated to $-\lambda$ is denoted by $k_{-\lambda}$ and is related to $\mathrm{H}^{0}(\lambda)$ under the $B$-equivariant projection map

$$
\begin{gathered}
\mathrm{p}_{\lambda}: \mathrm{H}^{0}(\lambda) \rightarrow k_{-\lambda}, \\
f \mapsto f\left(I_{n}\right) .
\end{gathered}
$$

When $\lambda$ and $\mu$ are both $B$-characters there is a natural multiplication map

$$
\mathrm{H}^{0}(\lambda) \otimes \mathrm{H}^{0}(\mu) \rightarrow \mathrm{H}^{0}(\lambda+\mu)
$$

It is a central fact (see e.g [1, Thm.3.1.2]) that this map is surjective in case $\lambda$ and $\mu$ are both dominant.

2.5. The fundamental representations. The modules $\mathrm{H}^{0}\left(\omega_{i}\right)$ induced by the fundamental characters $\omega_{i}, i=1, \ldots, n-1$, are called fundamental representations of $G$. The fundamental representations are simple $G$-modules. Moreover, $\mathrm{H}^{0}\left(\omega_{n-i}\right)$ is the module dual to $\mathrm{H}^{0}\left(\omega_{i}\right)$. For a fixed integer $i$ and a collection $\mathbf{a}=\left(a_{1}, a_{2}, \ldots, a_{i}\right)$ of increasing integers

$$
1<a_{1}<a_{2}<\cdots<a_{i} \leq n
$$

we let

$$
v_{\mathbf{a}}: G \rightarrow k
$$


denote the map which takes a matrix $g$ in $G$ to the minor determined by the row numbers $a_{1}, a_{2}, \ldots, a_{i}$ and the column numbers $1,2, \ldots, i$. Then $v_{\mathbf{a}}$ is easily seen to be an element of $\mathrm{H}^{0}\left(\omega_{i}\right)$. In fact, the collection of elements $v_{\mathbf{a}}$ form a basis for the fundamental representation $\mathrm{H}^{0}\left(\omega_{i}\right)$ (cf. (3) below). In this notation the lowest weight space of $\mathrm{H}^{0}\left(\omega_{i}\right)$ is generated by $v_{(1,2, \ldots, i)}$.

2.6. Exterior products. Let $V=k^{n}$ denote the standard representation of $G$ and let $V^{*}$ denote its dual. We let $e_{1}, \ldots, e_{n}$ denote the standard basis of the vector space $V=k^{n}$, and let $e_{1}^{*}, \ldots, e_{n}^{*}$ denote the dual basis of $V^{*}$. As a basis for the $i$-th exterior products $\wedge^{i} V$ we choose the set of elements

$$
e_{\mathbf{a}}=e_{a_{1}} \wedge \cdots \wedge e_{a_{i}} \in \wedge^{i} V
$$

where $\mathbf{a}=\left(a_{1}, \ldots, a_{i}\right)$ denotes an increasing sequence

$$
1 \leq a_{1}<a_{2}<\cdots<a_{i} \leq n,
$$

of integers. Similarly we consider

$$
e_{\mathbf{a}}^{*}=e_{a_{1}}^{*} \wedge \cdots \wedge e_{a_{i}}^{*} \in \wedge^{i} V^{*},
$$

as a basis of $\wedge^{i} V^{*}$. We consider $\wedge^{i} V^{*}$ and $\wedge^{i} V$ as dual vectorspaces by applying the map

$$
\begin{gathered}
\wedge^{i} V^{*} \otimes \wedge^{i} V \rightarrow k, \\
\left(f_{1} \wedge \cdots \wedge f_{i}\right) \otimes\left(v_{1} \wedge \cdots \wedge v_{i}\right) \rightarrow \operatorname{det}\left(f_{s}\left(v_{t}\right)\right)_{1 \leq s, t \leq i},
\end{gathered}
$$

In particular, the basis $\left\{e_{\mathbf{a}}^{*}\right\}$ of $\wedge^{i} V^{*}$ is dual to the basis $\left\{e_{\mathbf{a}}\right\}$ of $\wedge^{i} V$. We then identify $\mathrm{H}^{0}\left(\omega_{i}\right)$ with $\wedge^{i} V$ using the $G$-equivariant isomorphism

$$
\wedge^{i} V^{*} \stackrel{\simeq}{\rightarrow} \mathrm{H}^{0}\left(\omega_{i}\right)
$$

defined by

$$
\mathfrak{v} \mapsto\left(g \mapsto \mathfrak{v}\left(g e_{1} \wedge \cdots \wedge e_{i}\right)\right) .
$$

In particular, we identify $e_{\mathbf{a}}^{*}$ with $v_{\mathbf{a}}$.

2.7. The symplectic group. When $n=2 m$ is even we let $\bar{G}=$ $\mathrm{Sp}_{2 m}(k)$ denote the symplectic group of rank $m$. We consider $\bar{G}$ as the subgroup of elements $g$ in $G=\mathrm{SL}_{n}(k)$ which satisfies the relation

$$
g^{T} A g=A
$$

where $A$ denotes the matrix

$$
A=\left(\begin{array}{cc}
0 & I_{m} \\
-I_{m} & 0
\end{array}\right)
$$

and $I_{m}$ denotes the identity matrix of size $m$. The Borel subgroup of $\bar{G}$ consisting of the upper triangular matrices contained in $\bar{G}$ will be denoted by $\bar{B}$ while the unipotent part of $\bar{B}$ will be denoted by $\bar{U}$. Moreover, we let $\bar{U}^{-}$denote the set of lower triangular unipotent elements in $\bar{G}$. Finally we let $\bar{T}$ denote the maximal torus in $\bar{G}$ consisting of the set of diagonal matrices within $\bar{G}$. 
2.8. Symplectic weights, roots and fundamental characters. When $\lambda$ is a $T$-character we let $\bar{\lambda}$ denote its restriction to $\bar{T}$. The group $\bar{T}$ consists of the set of diagonal matrices in $G$ satisfying that the $i$-th diagonal entry, for $i=1, \ldots, n$, is the inverse of the $(n+1-i)$ th diagonal entry. In particular, $\bar{\epsilon}_{1}, \bar{\epsilon}_{2}, \ldots, \bar{\epsilon}_{m}$ form a basis for the group of characters $X^{*}(\bar{T})$ of $\bar{T}$. Moreover, any root $\epsilon_{i}-\epsilon_{j}$ of $G$ restricts to a root $\bar{\epsilon}_{i}-\bar{\epsilon}_{j}$ of $\bar{G}$; in particular this restriction is nonzero. The fundamental characters of $\bar{G}$ are the restrictions $\bar{\omega}_{i}, i=1, \ldots, m$, of the first $m$ fundamental characters for $G$.

We identify the characters of $\bar{B}$ with the characters of $\bar{T}$. Thus any $\bar{T}$-character $\bar{\lambda}$ defines an induced $\bar{G}$-module :

(5) $\operatorname{Ind} \frac{\bar{G}}{B}(\bar{\lambda})=\left\{f: \bar{G} \rightarrow k: f(g b)=\bar{\lambda}(b)^{-1} f(g)\right.$, for $\left.b \in \bar{B}, g \in \bar{G}\right\}$,

as in the case of $G$. We also use the short notation $\mathrm{H}^{0}(-\bar{\lambda})$ for the $\bar{G}$-module in (5). Then any $T$-character $\lambda$ defines a $\bar{G}$-equivariant restriction map

$$
\mathrm{H}^{0}(\lambda) \rightarrow \mathrm{H}^{0}(\bar{\lambda})
$$

As (positive) roots of $G$ restricts to (positive) roots of $\bar{G}$ the morphism (6) will map the lowest weight space (as a $G$-module) of $\mathrm{H}^{0}(\lambda)$ isomorphically to the lowest weight space of $\mathrm{H}^{0}(\bar{\lambda})$. In fact, any $\bar{T}$-weight $\bar{\mu}$ of $\mathrm{H}^{0}(\lambda)$ distinct from $-\bar{\lambda}$ must satisfy that $\bar{\mu}+\bar{\lambda}$ is a nonzero sum of positive roots of $\bar{G}$.

2.9. The Steinberg module. Let $\rho=\omega_{1}+\cdots+\omega_{n-1}$ denote the sum of the fundamental characters of $G$. The Steinberg module St is by definition the $G$-module $\mathrm{H}^{0}((p-1) \rho)$. The Steinberg module is an irreducible and self-dual $G$-module. Similarly we may define the Steinberg module in the symplectic case to be the $\bar{G}$-module $\overline{\mathrm{St}}$ induced from the $(p-1)$-th multiple of the sum $\bar{\omega}_{1}+\cdots+\bar{\omega}_{m}$, of the fundamental characters of $\bar{G}$. Also in this case $\overline{\text { St }}$ will be an irreducible and selfdual $\bar{G}$-module.

2.10. The dualizing sheaf. The dualizing sheaf $\omega_{G / B}$ on $G / B$ is a $G$ linearized line bundle on $G / B$ associated to the $B$-character $2 \rho$. As a consequence the set of global sections of a power $\omega_{G / B}^{N}$ of $\omega_{G / B}$ may be $G$-equivariantly identified with the induced module $\mathrm{H}^{0}(-2 N \rho)$. A similar result is true in the symplectic case once $\rho$ is substituted by the sum $\bar{\omega}_{1}+\cdots+\bar{\omega}_{m}$ of the fundamental characters. As we will see in the next section the case $N=1-p$ is related to the notion of Frobenius splittings.

\section{Frobenius sPlitting}

Let $X$ denote a variety. The absolute Frobenius morphism on $X$ is the map of schemes $F_{X}: X \rightarrow X$ which on the level of points is the 
identity map and as a map of structure sheaves

$$
F_{X}^{\sharp}: \mathcal{O}_{X} \rightarrow\left(F_{X}\right)_{*} \mathcal{O}_{X},
$$

is the $p$-th power map.

Definition 3.1. ([8]) The variety $X$ is said to admit a Frobenius splitting (or an F-splitting in short) if the map of sheaves

$$
F_{X}^{\sharp}: \mathcal{O}_{X} \rightarrow\left(F_{X}\right)_{*} \mathcal{O}_{X},
$$

admits an $\mathcal{O}_{X}$-linear splitting, i.e. an element

$$
s \in \operatorname{End}_{F}(X):=\operatorname{Hom}_{\mathcal{O}_{X}}\left(\left(F_{X}\right)_{*} \mathcal{O}_{X}, \mathcal{O}_{X}\right)
$$

such that the composition $s \circ F_{X}^{\sharp}$ is the identity map on $\mathcal{O}_{X}$.

3.1. A local description. When $X$ is a smooth variety we let $\omega_{X}$ denote the dualizing sheaf of $X$. By duality, for the finite morphism $F_{X}$, there is a canonical $k$-linear identification

$$
\operatorname{End}_{F}(X)^{(1)} \simeq \mathrm{H}^{0}\left(X, \omega_{X}^{1-p}\right),
$$

where we use the notation $\operatorname{End}_{F}(X)^{(1)}$ for the set $\operatorname{End}_{F}(X)$ with $k$ structure twisted by the $p$-th root map on $k$. In particular, we may think of elements in $\operatorname{End}_{F}(X)$ as global sections of a linebundle. For a point $P \in X$ we may describe the identification (8) locally as follows (see [8, Prop.5]): let $x_{1}, x_{2}, \ldots, x_{N}$ denote a system of local coordinates at a point $P$ of $X$. Then the section

$$
x_{1}^{a_{1}} x_{2}^{a_{2}} \cdots x_{N}^{a_{N}}\left(d x_{1} \wedge d x_{2} \wedge \cdots \wedge d x_{N}\right)^{1-p},
$$

of $\omega_{X}^{1-p}$ corresponds to the element in $\operatorname{End}_{F}(X)$ satisfying

$$
x_{1}^{b_{1}} x_{2}^{b_{2}} \cdots x_{N}^{b_{N}} \mapsto x_{1}^{\frac{a_{1}+b_{1}+1}{p}-1} x_{2}^{\frac{a_{2}+b_{2}+1}{p}-1} \cdots x_{n}^{\frac{a_{N}+b_{N}+1}{p}-1},
$$

where we consider the right hand side to be zero if one of the rational exponents $\frac{a_{i}+b_{i}+1}{p}-1$ is not an integer.

Example 3.2. Consider the case $X=\mathbb{A}^{N}$ in which $\omega_{X}$ is isomorphic to $\mathcal{O}_{X}$. Thus elements in $\operatorname{End}_{F}(X)$ correspond to polynomials in

$$
k[X]=k\left[X_{1}, \ldots, X_{N}\right] .
$$

This correspondence is uniquely determined up to nonzero constants. Hence we may speak of an element $g$ in $k\left[X_{1}, \ldots, X_{N}\right]$ as defining a Frobenius splitting of $\mathbb{A}^{N}$ (at least up to a nonzero constants). A necessary, but not sufficient, condition for this is that the monomial

$$
\left(X_{1} X_{2} \cdots X_{N}\right)^{p-1} \text {, }
$$

appears in $g$ with nonzero coefficient. To obtain a sufficient condition we need to add the conditions that all monomials of the form

$$
\left(X_{1} X_{2} \cdots X_{N}\right)^{p-1} M^{p}
$$

for $M$ a nonzero monomial in $k\left[X_{1}, \ldots, X_{N}\right]$, should not appear in $g$. 
3.2. Compatible splitting. Let $Y$ denote a closed subvariety of $X$ and let $\mathcal{J}_{Y}$ denote the associated sheaf of ideals in $\mathcal{O}_{X}$.

Definition 3.3. An element $s \in \operatorname{End}_{F}(X)$ is said to be compatible with $Y$ if

$$
s\left(\left(F_{X}\right)_{*} \mathcal{J}_{Y}\right) \subset \mathcal{J}_{Y} .
$$

If, moreover, $s$ defines a Frobenius splitting of $X$ then we say that $Y$ is compatibly Frobenius split in $X$. The set of elements in $\operatorname{End}_{F}(X)$ which are compatible with $Y$ will be denoted by $\operatorname{End}_{F}(X, Y)$.

3.3. Multiplicities. When $X$ and $Y$ are both smooth varieties we may use the local description in (8) to obtain a criterion for the compatibility of $Y$ : let $P \in Y$ and choose a system of local coordinates at $P$ in such a way that the ideal of $Y$ is generated by $x_{1}, \ldots, x_{d}$ (with $d$ denoting the codimension of $Y$ in $X$ ). Then the element (9) will map the ideal $\left(x_{1}, \ldots, x_{d}\right)$ to itself if one of the following conditions are satisfied

- $a_{i} \geq p$ for some $1 \leq i \leq d$.

- $a_{1}=a_{2}=\cdots=a_{d}=p-1$.

In particular, this is the case if (9) vanishes with multiplicity at least $(p-1) d$ along $Y$. This leads to the following definition

Definition 3.4. Let $X$ be a smooth variety and let $Y$ denote a closed smooth subvariety. We say that an element $s$ of $\operatorname{End}_{F}(X)$ vanishes with multiplicity $m$ along $Y$, if the same is true for the associated global section of the line bundle $\omega_{X}^{1-p}$.

It then follows

Lemma 3.5. Assume that $X$ and $Y$ are smooth varieties and that $Y$ is of codimension $d$ in $X$. Let $s$ be an element of $\operatorname{End}_{F}(X)$ vanishing with multiplicity at least $d(p-1)$ along $Y$. Then $s$ is compatible with $Y$.

When $s$ is assumed to be a Frobenius splitting of $X$ a vanishing multiplicity along $Y$ of size $d(p-1)$ is said to be maximal. This notion is explained by the following result

Proposition 3.6. (44, Sect.2]) Let $X$ be a smooth variety and let $Y$ denote a smooth closed subvariety of codimension $d$. Let $s$ denote a Frobenius splitting of $X$. Then $s$ vanishes with multiplicity at most $(p-1) d$ along $Y$. Moreover, if s vanishes with maximal multiplicity then the blow-up of $X$ at $Y$ admits a Frobenius splitting which is compatible with the exceptional divisor.

The maximal vanishing multiplicty is particular important in the case of the diagonal subvariety $\operatorname{diag}(X)$ in the product $X \times X$. With $\triangle$ denoting the exterior tensorproduct on $X \times X$ we have the following result 
Theorem 3.7. Let $X$ denote a smooth projective variety. Let $\mathcal{L}$ denote an ample line bundle on $X$, and $\mathcal{M}_{1}$ and $\mathcal{M}_{2}$ denote globally generated line bundles on $X$. Define $\mathcal{L}_{i}=\mathcal{L} \otimes \mathcal{M}_{i}$, for $i=1,2$. Let further $\mathcal{J}$ denote the sheaf of ideals defining the diagonal subvariety $\operatorname{diag}(X)$ in $X \times X$. If $X \times X$ admits a Frobenius splitting vanishing with maximal multiplicity along the diagonal, then the natural restriction map

$$
\mathrm{H}^{0}\left(X \times X, \mathcal{J} \otimes\left(\mathcal{L}_{1} \otimes \mathcal{L}_{2}\right)\right) \rightarrow \mathrm{H}^{0}\left(X, \Omega_{X}^{1} \otimes \mathcal{L}_{1} \otimes \mathcal{L}_{2}\right),
$$

is surjective.

Theorem 3.7 was proved in [4, Sect.3] in the case of a generalized flag variety $X$. The general form of Theorem 3.7 was obtained in [6, Thm.6.1]. Notice that when $X$ is a generalized flag variety (in fact, any Schubert variety [6, Sect.6])) then any pair $\mathcal{L}_{1}, \mathcal{L}_{2}$ of ample line bundles on $X$ will be of the form described in Theorem 3.7. In particular, in this case Wahl's conjecture is a consequence of Theorem 3.7.

As a consequence of Proposition 3.6 the existence of a Frobenius splitting of $X \times X$ vanishing with maximal multiplicty along the diagonal will imply that the projectivized tangent bundle of $X$ admits a Frobenius splitting. We therefore recall the following vanishing result

Theorem 3.8. ([6, Prop.6.8]) Let $X$ denote a smooth projective variety and assume that the projectivized tangent bundle on $X$ admits a Frobenius splitting. Let $\mathcal{L}$ (resp. $\mathcal{M}$ ) denote a very ample (resp. globally generated) line bundle on $X$ and let $j>0$ denote an integer. Then

$$
\mathrm{H}^{i}\left(Z, S^{j} \Omega_{Z} \otimes \mathcal{L}^{2 j} \otimes \mathcal{M}\right)=0, \text { for } i>0 .
$$

We refer to [6] for further related vanishing results.

3.4. The evaluation map. Any element in $\operatorname{End}_{F}(X)$ may be evaluated at the constant function $1 \in k[X]$. This defines a map

$$
\begin{gathered}
\operatorname{ev}_{\mathrm{X}}: \operatorname{End}_{F}(X) \rightarrow k[X], \\
s \mapsto s(1) .
\end{gathered}
$$

Thus $s$ in $\operatorname{End}_{F}(X)$ defines a Frobenius splitting of $X$ if and only if $\operatorname{ev}_{X}(s)=1$. In particular, the condition that $s$ is a Frobenius splitting may be checked on an open dense subset of $X$; i.e. if $V$ is an open dense subset of $X$ and if the restriction of $s$ to $V$ defines a Frobenius splitting of $V$, then $s$ defines a Frobenius splitting of $X$. In case $k[X]=k$, e.g. if $X$ is complete and irreducible, any element $s \in \operatorname{End}_{F}(X)$ with nonzero evaluation $\operatorname{ev}_{X}(s)$ will thus define a Frobenius splitting of $X$ up to a nonzero constant.

When $X$ is smooth we may use the evaluation map $\mathrm{ev}_{X}$ and the identification (8) to define a $k$-linear map ( $k$ is perfect)

$$
\mathrm{H}^{0}\left(X, \omega_{X}^{1-p}\right) \rightarrow k \text {. }
$$


This map will, by abuse of notation, also be denoted by $\mathrm{ev}_{X}$ and will also be called the evaluation map. By the local description (9) we may (locally) consider $\mathrm{ev}_{X}$ as the map which picks out the coefficient in front of

$$
\left(x_{1} x_{2} \cdots x_{N}\right)^{p-1}\left(d x_{1} \wedge \cdots \wedge d x_{N}\right)^{1-p},
$$

in the local monomial expansions of an element $s \in \mathrm{H}^{0}\left(X, \omega_{X}^{1-p}\right)$ relative to a system of local coordinates $x_{1}, \ldots, x_{N}$ at a point $P$ of $X$. In particular, $\operatorname{ev}_{X}(s)$ is nonzero if this coefficient is nonzero for some (and hence any) point $P$.

3.5. Canonical Frobenius splittings. Let now $X$ denote a $G$-variety, and consider the vector space $\operatorname{End}_{F}(X)$ as a $G$-module in the natural way. A $G$-equivariant morphism

$$
\phi: \mathrm{St} \otimes \mathrm{St} \rightarrow \operatorname{End}_{F}(X)^{(1)},
$$

is said to be a canonical Frobenius splitting of $X$ if the image of $\phi$ contains a Frobenius splitting of $X$. The notion of a canonical Frobenius splitting of a $\bar{G}$-variety is defined similarly.

\section{Residual nORMal CROSSing}

In the following we will consider a polynomial ring $k\left[X_{1}, \ldots, X_{N}\right]$ and a totally ordered subset $J_{1}$ of the set of variables $X_{i}$. The order on $J_{1}$ will be denoted by $\leq_{1}$.

Definition 4.1. An element $g$ in $k\left[X_{1}, \ldots, X_{N}\right]$ is said to be a residual normal crossing relative to the ordered subset $J_{1}$, if the following recursive conditions are satisfied

(1) If $J_{1}$ is empty then $g$ is a nonzero constant.

(2) If $J_{1}$ is nonempty and $X_{j}$ is the minimal element in $J_{1}$ then $X_{j}$ divides $g$. Moreover, the evaluation $\left(\frac{g}{X_{j}}\right)_{\mid X_{j}=0}$ of the quotient $\frac{g}{X_{j}}$ at $X_{j}=0$, is then a residual normal crossing relative to the subset $J_{1} \backslash\left\{X_{j}\right\}$, with the induced total order.

Notice that a necessary (but not sufficient) condition for $g$ to be a residual normal crossing relative to $J_{1}$, is that the monomial

$$
\prod_{j \in J_{1}} X_{j}
$$

appears in $g$ with nonzero coefficient. In fact, even more general results like this are true (see Proposition 4.4 below).

When $\left(J_{1}, \leq_{1}\right)$ and $\left(J_{2}, \leq_{2}\right)$ denote disjoint totally ordered subsets of the set of variables, then we let $\leq_{1,2}$ denote the total order on the union $J_{1} \cup J_{2}$ defined by : $X_{a} \leq_{1,2} X_{b}$ if one of the following conditions are satisfied

- $X_{a}, X_{b} \in J_{1}$ and $X_{a} \leq_{1} X_{b}$.

- $X_{a}, X_{b} \in J_{2}$ and $X_{a} \leq_{2} X_{b}$. 
- $X_{a} \in J_{1}$ and $X_{b} \in J_{2}$.

This explains the notation in the following statement

Lemma 4.2. Let $f$ and $g$ denote elements in $k\left[X_{1}, \ldots, X_{N}\right]$. Assume that $g$ is a residual normal crossing relative to a subset $J_{1}$, and that the evaluation

$$
\bar{f}=f_{\mid\left\{X_{j}=0: X_{j} \in J_{1}\right\}}
$$

is a residual normal crossing relative to an ordered subset $J_{2}$. Then $J_{1}$ and $J_{2}$ are disjoint and the product $f g$ is a residual normal crossing relative to the union $J_{1} \cup J_{2}$ ordered by $\leq_{1,2}$.

Proof. That $J_{1}$ and $J_{2}$ are disjoint is clear as $\bar{f}$ does not involve any of the variables in $J_{1}$. In particular, the monomial

$$
\prod_{X_{j} \in J_{2}} X_{j}
$$

could not appear in $\bar{f}$ unless $J_{1}$ and $J_{2}$ were disjoint. That $f g$ is a residual normal crossing relative to the ordered subset $J_{1} \cup J_{2}$ now follows directly from the recursive definition.

The definition of being a residual normal crossing does not involve any of the variables $X_{j}$ outside $J_{1}$. In particular, we have the following easy, but still useful, observation

Lemma 4.3. Let $g \in k\left[X_{1}, \ldots, X_{N}\right]$ be a residual normal crossing relative to an ordered subset $J_{1}$. Assume that $X_{j} \notin J_{1}$ and let

$$
h=g\left(X_{1}, \cdots, X_{j-1}, f, X_{j+1}, \cdots, X_{N}\right),
$$

denote effect of substituting the $j$-th variable in $g$ by a polynomial $f \in$ $k\left[X_{1}, \ldots, X_{N}\right]$. Then $h$ is also a residual normal crossing relative to the subset $J_{1}$.

4.1. Frobenius splitting and residual normal crossing. Consider a complete variety $X$ containing an open dense subset $V$ isomorphic to $\mathbb{A}^{N}$. As explained in Section 3.4 an element $s$ of $\operatorname{End}_{F}(X)$ defines a Frobenius splitting of $X$, up to a nonzero constant, if and only if the evaluation $\operatorname{ev}_{X}(s)$ is nonzero. Moreover, this condition may be checked locally and means that $s$, when expressed in a system of local coordinates $x_{1}, \ldots, x_{N}$ at a point $P$, contains the monomial

$$
\left(x_{1} x_{2} \cdots x_{N}\right)^{p-1}\left(d x_{1} \wedge \cdots \wedge d x_{N}\right)^{1-p},
$$

with nonzero coefficient. E.g. we could take $P$ to denote the origin (or any other point) in $V=\mathbb{A}^{N}$. In this case we may also formulate the nonzeroness condition of $\operatorname{ev}_{X}(s)$ as follows: consider, as explain in Example 3.2, the restriction $s_{\mid V}$, of $s$ to $V$, as an element in the coordinate ring

$$
k[V]=k\left[X_{1}, X_{2}, \ldots, X_{N}\right],
$$


of $V$. Then the monomial

$$
\left(X_{1} X_{2} \cdots X_{N}\right)^{p-1}
$$

should appear in $s_{\mid V}$ with nonzero coefficient. A condition like the latter may be checked using the notion of residual normal crossings. This is explained by the following result which follows easily from the definition.

Proposition 4.4. Let $g \in k\left[X_{1}, \ldots, X_{N}\right]$ denote a residual normal crossing relative to an ordered subset $J_{1}$ of the variables. Let d denote any positive integer. Then the monomial

$$
\prod_{X_{j} \in J_{1}} X_{j}^{d}
$$

appears with nonzero coefficient in $g^{d}$.

\section{Concrete Residual normal CROssings}

In this section we will study certain residual normal crossings associated to the group $U^{-}$. We start by observing that $U^{-}$is isomorphic to affine $\left(\begin{array}{l}n \\ 2\end{array}\right)$-space, and choose coordinate functions $Z_{i, j}, 1 \leq j<i \leq n$, in the natural way.

The functions $v_{\mathbf{a}}$ on $G$ defined in Section 2.5 are semi-invariant under translation by $B$ from the right and hence they are uniquely determined by their restrictions to $U^{-}$. It thus makes sense to consider $v_{\mathbf{a}}$ as functions on $U^{-}$; i.e. as elements in the polynomial ring $k\left[Z_{i, j}\right]_{1 \leq j<i \leq n}$. In the following we will consider the functions $(1 \leq r \leq n)$

$$
\mathfrak{f}_{r}=\prod_{s=1}^{r} v_{\left(\left\lfloor\frac{s}{2}\right\rfloor+1,\left\lfloor\frac{s}{2}\right\rfloor+2, \ldots, s\right)} \in k\left[U^{-}\right],
$$

where $\left\lfloor\frac{s}{2}\right\rfloor$ denotes the biggest integer smaller than $\frac{s}{2}$. We claim that $\mathfrak{f}_{r}$ is a residual normal crossing relative to an ordered subset of the variables $Z_{i, j}$. To make this precise we introduce the following ordering of the variables: $Z_{i, j} \leq Z_{i^{\prime}, j^{\prime}}$ if either $i+j<i^{\prime}+j^{\prime}$ or if $i+j=i^{\prime}+j^{\prime}$ and $i \leq i^{\prime}$. In the following any subset of the variables will, unless otherwise stated, be assumed to be ordered using this order.

Lemma 5.1. The polynomial $\mathfrak{f}_{r}$ is a residual normal crossing relative to the subset

of the variables.

$$
\left\{Z_{i, j}: i+j \leq r+1\right\}
$$

Proof. We proceed by induction in $r$. As

$$
\mathfrak{f}_{1}=1,
$$

the case $r=1$ is clear. Now assume that $1<r \leq n$ and that the statement is correct for $r-1$. Notice then

$$
\mathfrak{f}_{r}=\mathfrak{f}_{r-1} \cdot v_{\left(\left\lfloor\frac{r}{2}\right\rfloor+1, \ldots, r\right)},
$$


and that $v_{\left(\left\lfloor\frac{r}{2}\right\rfloor+1, \ldots, r\right)}$, when evaluated at $Z_{i, j}=0$ for $i+j \leq r$, equals

$$
\prod_{\substack{1 \leq j<i \leq n, i+j=r+1}} Z_{i, j}
$$

up to a sign. We may thus apply Lemma 4.2 to conclude that $\mathfrak{f}_{r}$ is a residual normal crossing relative to $Z_{i, j}$, for $i+j \leq r+1$. This ends the proof.

Let $\mathfrak{w}_{0}$ in $\mathrm{GL}_{n}(k)$ denote the anti-diagonal matrix whose $(i, j)$-th entry equals 1 if $i+j=n+1$ and equals 0 otherwise. Then

$$
\begin{gathered}
\tau: \mathrm{SL}_{n}(k) \rightarrow \mathrm{SL}_{n}(k), \\
\tau(g)=\mathfrak{w}_{0} g^{T} \mathfrak{w}_{0},
\end{gathered}
$$

defines an automorphism of the variety $G$ of order two which leaves $U^{-}$ invariant. The map $\tau$ is not a group automorphism but composing $\tau$ with the inverse map

$$
\iota: G \rightarrow G, \iota(g)=g^{-1},
$$

we obtain an involution $\sigma=\tau \circ \iota$ of $G$ leaving $B$ invariant. In the following we let $\mathfrak{g}_{r} \in k\left[U^{-}\right]$denote the composition $\mathfrak{f}_{r} \circ \tau$.

Lemma 5.2. The polynomial $\mathfrak{g}_{r}$ is a residual normal crossing relative to the reverse order on the subset

$$
\left\{Z_{i, j}: i+j \geq 2 n+1-r\right\}
$$

of the variables.

Proof. This follows directly from Lemma 5.1 once it is observed that the effect of $\tau$ on $k\left[U^{-}\right]$is given by the order reversing map $Z_{i, j} \mapsto$ $Z_{n+1-j, n+1-i}$.

Proposition 5.3. There exists a total order on the full set of variables $Z_{i, j}, 1 \leq j<i \leq n$, such that

$$
\mathfrak{f}_{n} \mathfrak{g}_{n-1}
$$

is a residual normal crossing relative to this ordered set.

Proof. This follows by applying Lemma 5.1, Lemma 5.2 and Lemma 4.2 .

Remark 5.4. In [4] a Frobenius splitting of $G / B$ vanishing with maximal multiplicity along the point eB was constructed. The restriction of this Frobenius splitting to $U^{-}$coincides with the $(p-1)$-th power of the composition $\left(\mathfrak{f}_{n} \mathfrak{g}_{n-1}\right) \circ \iota$ (see [4, Remark $A_{n}(\mathrm{i})$ ] and Lemma 6.2 below). The idea of the present paper is to induce this Frobenius splitting into a Frobenius splitting of $G / B \times G / B$ vanishing with maximal multiplicity along the diagonal. A similar remark applies in the symplectic case. 


\section{TENSORPRODUCTS OF FUNDAMENTAL REPRESENTATIONS}

In this section we will consider tensorproducts of the fundamental representations of $G$. To simplify notation we let $\omega_{n}$ and $\omega_{0}$ denote the trivial $T$-characters. Fix integers $0 \leq i, j \leq n$, and let $[i+j]_{n}$ denote either $i+j$ if $i+j \leq n$, and $i+j-n$ otherwise. We want to construct a $G$-equivariant nonzero morphism

$$
\eta_{i, j}: \mathrm{H}^{0}\left(\omega_{[i+j]_{n}}\right) \rightarrow \mathrm{H}^{0}\left(\omega_{i}\right) \otimes \mathrm{H}^{0}\left(\omega_{j}\right) .
$$

We divide this construction into to cases

6.1. The case $i+j \leq n$. By (3) it suffices to construct a nonzero $G$-equivariant morphism

$$
\wedge_{i, j}: \wedge^{i+j} V^{*} \rightarrow \wedge^{i} V^{*} \otimes \wedge^{j} V^{*} .
$$

The latter is defined to be the dual of the product map

$$
\wedge^{i} V \otimes \wedge^{j} V \rightarrow \wedge^{i+j} V
$$

With notation as in Section 2.5 and Section 2.6 it is then an easy exercise to check that

$$
\eta_{i, j}\left(v_{\mathbf{a}}\right)=\sum_{\gamma \in \mathrm{Sh}_{i, j}} \operatorname{sgn}(\gamma) v_{\left(a_{\gamma(1)}, \cdots, a_{\gamma(i)}\right)} \otimes v_{\left(a_{\gamma(i+1)}, \cdots, a_{\gamma(i+j)}\right)},
$$

where we use the notation $\mathrm{Sh}_{i, j}$ to denote the set of $(i, j)$-shuffles, i.e. the permutations $\gamma$ of $1,2 \ldots, i+j$ satisfying $\gamma(1)<\gamma(2)<\cdots<\gamma(i)$ and $\gamma(i+1)<\gamma(i+2)<\cdots<\gamma(i+j)$. In particular, we note

Lemma 6.1. The composition of $\eta_{i, j}$, for $i+j \leq n$, with the $B$ equivariant map

$$
\mathrm{p}_{\omega_{i}}: \mathrm{H}^{0}\left(\omega_{i}\right) \rightarrow k_{-\omega_{i}},
$$

defines a B-equivariant morphism

$$
\mathrm{H}^{0}\left(\omega_{i+j}\right) \rightarrow k_{-\omega_{i}} \otimes \mathrm{H}^{0}\left(\omega_{j}\right),
$$

given by

$$
v_{\mathbf{a}} \mapsto \begin{cases}1 \otimes v_{\left(a_{i+1}, \ldots, a_{i+j}\right)} & \text { if } a_{s}=s \text { for } s=1, \ldots, i, \\ 0 & \text { else, }\end{cases}
$$

when $\mathbf{a}=\left(a_{1}, \ldots, a_{i+j}\right)$.

Proof. This follows from (15) once it is observed that the natural morphism (16) is given by

$$
v_{\mathbf{b}} \mapsto \begin{cases}1 & \text { if } \mathbf{b}=(1,2, \ldots, \mathrm{i}), \\ 0 & \text { else, }\end{cases}
$$

for $\mathbf{b}=\left(b_{1}, \ldots, b_{i}\right)$. 
6.2. The case $i+j>n$. To define $\eta_{i, j}$ when $i+j>n$ we will use the involution $\sigma$ of $G$ defined in Section [5. Any element $f: G \rightarrow k$ in $\mathrm{H}^{0}\left(\omega_{s}\right)$ will by composition $f \circ \sigma$ with $\sigma$ define an element in $\mathrm{H}^{0}\left(\omega_{n-s}\right)$. This way we obtain a bijection between $\mathrm{H}^{0}\left(\omega_{s}\right)$ and $\mathrm{H}^{0}\left(\omega_{n-s}\right)$. Alternatively this may be formulate by saying that $\sigma$ defines a $G$-equivariant isomorphism

$$
\mathrm{H}^{0}\left(\omega_{s}\right)^{\sigma} \simeq \mathrm{H}^{0}\left(\omega_{n-s}\right),
$$

where $\mathrm{H}^{0}\left(\omega_{s}\right)^{\sigma}$ denotes the $G$-module which as a vector space is $\mathrm{H}^{0}\left(\omega_{s}\right)$ but where the $G$-action is twisted by $\sigma$. As $\eta_{n-i, n-j}$ has already been defined above we may now define

$$
\eta_{i, j}: \mathrm{H}^{0}\left(\omega_{i+j-n}\right) \rightarrow \mathrm{H}^{0}\left(\omega_{i}\right) \otimes \mathrm{H}^{0}\left(\omega_{j}\right),
$$

to the $\sigma$-twist of $\eta_{n-i, n-j}$.

In the following we will need more specific information about the structure of (17). Recall that $\iota$ denotes the map $\iota(g)=g^{-1}$ for $g \in U^{-}$. Then

Lemma 6.2. Let $2 \leq i \leq n$ denote an integer. Then the functions

$$
v_{\left(\left\lfloor\frac{i}{2}\right\rfloor+1,\left\lfloor\frac{i}{2}\right\rfloor+2, \ldots, i\right)} \circ \iota,
$$

and

$$
v_{\left(\left\lceil\frac{i}{2}\right\rceil+1,\left\lceil\frac{i}{2}\right\rceil+2, \ldots, i\right)},
$$

on $U^{-}$coincides up to a nonzero constant.

Proof. By concentrating on the first $i$ rows and columns we may assume that $i=n$. Now notice that

$$
v_{\left(\left\lceil\frac{n}{2}\right\rceil+1,\left\lceil\frac{n}{2}\right\rceil+2, \ldots, n\right)},
$$

is a $B \times B$-semiinvariant functions in $\mathrm{H}^{0}\left(\omega_{\left\lfloor\frac{n}{2}\right\rfloor}\right)$; in particular, it must generate the highest weight space in $\mathrm{H}^{0}\left(\omega_{\left\lfloor\frac{n}{2}\right\rfloor}\right)$. Similarly, $v_{\left(\left\lfloor\frac{n}{2}\right\rfloor+1,\left\lfloor\frac{n}{2}\right\rfloor+2, \ldots, n\right)}$ is a a highest weight vector of $\mathrm{H}^{0}\left(\omega_{\left\lceil\frac{n}{2}\right\rceil}\right)$ and thus by (17) the composition

$$
v_{\left(\left\lfloor\frac{n}{2}\right\rfloor+1,\left\lfloor\frac{n}{2}\right\rfloor+2, \ldots, n\right)} \circ \sigma,
$$

is a highest weight vector in $\mathrm{H}^{0}\left(\omega_{\left\lfloor\frac{n}{2}\right\rfloor}\right)$. It follows that (19) and (20) coincide up to a nonzero constant as functions on $G$. To end the proof observe that (20) coincide with (18) (for $i=n$ ) as functions on $G$ and thus also on $U^{-}$.

Remark 6.3. As observed by Shrawan Kumar the above described maps $\eta_{i, j}$ are also predicted by the PRVK-conjecture. E.g. when $i+j \leq$ $n-1$, the PRVK-conjecture (see e.g. [1, Thm4.3.2]) predicts the existence of a unique map (up to constants)

$$
\mathrm{H}^{0}(\theta) \rightarrow \mathrm{H}^{0}\left(\omega_{i}\right) \otimes \mathrm{H}^{0}\left(\omega_{j}\right),
$$


where $\theta$ is the dominant T-characters in the Weyl group orbit of the character $\omega_{i}+w_{0} \omega_{j}$ ( $w_{0}$ denotes the element of maximal length in the Weyl group). It is an easy exercise to check that $\theta$ coincide with $\omega_{i+j}$.

\section{VANishing BehaViour of ELEMENTS in the IMAge of $\eta_{i, j}$}

Elements in the image of $\eta_{i, j}$ may be regarded as regular functions on $G \times G$. In this section we will obtain a lower bound on their order of vanishing along the $\operatorname{diagonal} \operatorname{diag}(G)$ of $G \times G$. We let $g, h \in G$ denote elements with entries $g_{s, t}$ and $h_{s, t}, 1 \leq s, t \leq n$, respectively, and form the $n \times 2 n$ matrix

$$
M(g, h)=\left(\begin{array}{cccccc}
g_{1,1} & \cdots & g_{1, n} & h_{1,1} & \cdots & h_{1, n} \\
g_{2,1} & \cdots & g_{2, n} & h_{2,1} & \cdots & h_{2, n} \\
\vdots & \cdots & \vdots & \vdots & \cdots & \vdots \\
g_{n, 1} & \cdots & g_{n, n} & h_{n, 1} & \cdots & h_{n, n}
\end{array}\right) .
$$

7.1. The case $i+j \leq n$. Fix $i$ and $j$ such that $i+j \leq n$ and an increasing sequence $\mathbf{a}=\left(a_{1}, \ldots, a_{i+j}\right)$.

Lemma 7.1. The evaluation of the function $\eta_{i, j}\left(v_{\mathbf{a}}\right)$ at $(g, h)$ coincides with the $(i+j)$-minor of $M(g, h)$ determined by the row numbers

$$
a_{1}, a_{2} \ldots, a_{i+j}
$$

and the column numbers

$$
1, \ldots, i \text { and } n+1, \ldots, n+j .
$$

In particular, the element $\eta_{i, j}\left(v_{\mathbf{a}}\right)$ vanishes with multiplicity at least $\min (i, j)$ along the diagonal in $G \times G$.

Proof. Recall that $v_{\mathbf{b}}$, for $\mathbf{b}=\left(b_{1}, \ldots, b_{s}\right)$, is defined as the map $G \rightarrow k$ which takes a matrix to its $s \times s$-minor determined by the row numbers $b_{1}, \ldots, b_{s}$ and the column numbers $1, \ldots, s$. Thus, by the Laplace expansion theorem, we recognize the right hand side of (15) as the determinant of the square matrix

$$
\left(\begin{array}{cccccc}
g_{a_{1}, 1} & \cdots & g_{a_{1}, i} & h_{a_{1}, 1} & \cdots & h_{a_{1}, j} \\
g_{a_{2}, 1} & \cdots & g_{a_{2}, i} & h_{a_{2}, 1} & \cdots & h_{a_{2}, j} \\
\vdots & \cdots & \vdots & \vdots & \cdots & \vdots \\
g_{a_{i+j}, 1} & \cdots & g_{a_{i+j}, i} & h_{a_{i+j}, 1} & \cdots & h_{a_{i+j}, j}
\end{array}\right) .
$$

This ends the proof of the first part of the statement.

For the vanishing part of the statement we may, by symmetry, assume that $i \leq j$. On the diagonal (i.e. when $g=h$ ) the matrix $M(g, h)$ contains $i$ pairs of equal columns; column 1 and $i+1$, column 2 and $i+2$ etc. In particular, if we subtract column $s$ from column $n+s$, for $s=1, \ldots, i$, before calculating the $(i+j)$-minor (22), then the vanishing statement is readily apparent. 
7.2. The case $i+j>n$. Now fix $i$ and $j$ such that $i+j>n$ and recall that $\eta_{i, j}$ was defined using the $\sigma$-twist of $\eta_{n-i, n-j}$. Then Lemma 7.1 directly implies

Lemma 7.2. Every element within the image of $\eta_{i, j}$ will vanish with multiplicity at least $\min (n-i, n-j)$ along the diagonal in $G \times G$.

\section{Frobenius SPlitting of $G / B \times G / B$}

In this section we will construct a canonical Frobenius splitting

$$
\eta: \mathrm{St} \otimes \mathrm{St} \rightarrow \operatorname{End}_{F}(G / B \times G / B)^{(1)},
$$

of $G / B \times G / B$ where any element in the image will vanish with multiplicity at least $(p-1) \operatorname{dim}(G / B)$ along the diagonal. In particular, any element in the image of $\eta$ will be compatible with the diagonal $\operatorname{diag}(G / B)$. Actually we will construct $\eta$ using the $G$-equivariant identification

$$
\operatorname{End}_{F}(G / B \times G / B)^{(1)} \simeq \mathrm{H}^{0}(2(p-1) \rho) \otimes \mathrm{H}^{0}(2(p-1) \rho),
$$

arising from the remarks in Section 2.10

8.1. Vanishing multiplicities. The map $\eta$ is constructed as the tensorproduct of a collection of the maps $\eta_{i, j}$. To describe this construction we start by considering the tensorproduct of the maps $(1 \leq s<n)$

$$
\eta_{\left\lfloor\frac{s}{2}\right\rfloor,\left\lceil\frac{s}{2}\right\rceil}: \mathrm{H}^{0}\left(\omega_{s}\right) \rightarrow \mathrm{H}^{0}\left(\omega_{\left\lfloor\frac{s}{2}\right\rfloor}\right) \otimes \mathrm{H}^{0}\left(\omega_{\left\lceil\frac{s}{2}\right\rceil}\right),
$$

where $\left\lfloor\frac{s}{2}\right\rfloor$ (resp. $\left\lceil\frac{s}{2}\right\rceil$ ) denotes the largest (resp. smallest) integer less (resp. larger) than $\frac{s}{2}$. This way we obtain a $G$-equivariant map

$$
\bigotimes_{s=1}^{n-1} \mathrm{H}^{0}\left(\omega_{s}\right) \rightarrow \bigotimes_{s=1}^{n-1}\left(\mathrm{H}^{0}\left(\omega_{\left\lfloor\frac{s}{2}\right\rfloor}\right) \otimes \mathrm{H}^{0}\left(\omega_{\left\lceil\frac{s}{2}\right\rceil}\right)\right) .
$$

Next we define

$$
\bigotimes_{s=1}^{n-1} \mathrm{H}^{0}\left(\omega_{s}\right) \rightarrow \bigotimes_{s=1}^{n-1}\left(\mathrm{H}^{0}\left(\omega_{\left\lfloor\frac{s+n}{2}\right\rfloor}\right) \otimes \mathrm{H}^{0}\left(\omega_{\left\lceil\frac{s+n}{2}\right\rceil}\right)\right)
$$

as the tensorproduct of the maps $(1 \leq s<n)$

$$
\eta_{\left\lfloor\frac{s+n}{2}\right\rfloor,\left\lceil\frac{s+n}{2}\right\rceil}: \mathrm{H}^{0}\left(\omega_{s}\right) \rightarrow \mathrm{H}^{0}\left(\omega_{\left\lfloor\frac{s+n}{2}\right\rfloor}\right) \otimes \mathrm{H}^{0}\left(\omega_{\left\lceil\frac{s+n}{2}\right\rceil}\right) .
$$

We then notice

Lemma 8.1. Any element in the image of (24) or (25) will vanish with multiplicity at least $\frac{n^{2}-2 n}{4}$, when $n$ is even, and at least $\frac{(n-1)^{2}}{4}$, when $n$ is odd, along the diagonal in $G \times G$.

Proof. For the map (24) the statement follows from Lemma 7.1 and the formula

$$
\sum_{s=1}^{n-1}\left\lfloor\frac{s}{2}\right\rfloor= \begin{cases}\frac{(n-1)^{2}}{4} & \text { when } n \text { odd } \\ \frac{n^{2}-2 n}{4} & \text { when } n \text { even. }\end{cases}
$$


For (25) we apply Lemma 7.2 along with (26)

\subsection{The construction of $\eta$.}

Lemma 8.2. There exists a nonzero G-equivariant map

$$
\mathrm{St} \rightarrow \bigotimes_{s=1}^{n-1} \mathrm{H}^{0}\left(\omega_{s}\right)^{\otimes(p-1)},
$$

which is uniquely determined up to nonzero constants.

Proof. As $\mathrm{H}^{0}\left(\omega_{s}\right)$ and $\mathrm{H}^{0}\left(\omega_{n-s}\right)$, for $s=1, \ldots, n-1$, are dual $G$ modules we start by observing that both sides of (27) are self-dual modules. Hence, maps of the form (27) are in 1-1 correspondence with nonzero $G$-equivariant maps

$$
\bigotimes_{s=1}^{n-1} \mathrm{H}^{0}\left(\omega_{s}\right)^{\otimes(p-1)} \rightarrow \text { St. }
$$

By Frobenius reciprocity the latter maps are constant multiples of the product map. This ends the proof.

We may now combine (27) with the $(p-1)$-th tensorpower of (24) and (25). This leads to $G$-equivariant maps

$$
\eta_{1}: \mathrm{St} \rightarrow \bigotimes_{s=1}^{n-1}\left(\mathrm{H}^{0}\left(\omega_{\left\lfloor\frac{s}{2}\right\rfloor}\right) \otimes \mathrm{H}^{0}\left(\omega_{\left\lceil\frac{s}{2}\right\rceil}\right)\right)^{\otimes(p-1)},
$$

and

$$
\eta_{2}: \mathrm{St} \rightarrow \bigotimes_{s=1}^{n-1}\left(\mathrm{H}^{0}\left(\omega_{\left\lfloor\frac{s+n}{2}\right\rfloor}\right) \otimes \mathrm{H}^{0}\left(\omega_{\left\lceil\frac{s+n}{2}\right\rceil}\right)\right)^{\otimes(p-1)}
$$

Next we take the tensorproduct of $\eta_{1}$ and $\eta_{2}$ with the $(p-1)$-th tensorpower of

$$
\eta_{\left\lfloor\frac{n}{2}\right\rfloor,\left\lceil\frac{n}{2}\right\rceil}: k \rightarrow \mathrm{H}^{0}\left(\omega_{\left\lfloor\frac{n}{2}\right\rfloor}\right) \otimes \mathrm{H}^{0}\left(\omega_{\left\lceil\frac{n}{2}\right\rceil}\right),
$$

and arrive at a $G$-equivariant map

$$
\mathrm{St} \otimes \mathrm{St} \rightarrow \bigotimes_{s=1}^{2 n-1}\left(\mathrm{H}^{0}\left(\omega_{\left\lfloor\frac{s}{2}\right\rfloor}\right) \otimes \mathrm{H}^{0}\left(\omega_{\left\lceil\frac{s}{2}\right\rceil}\right)\right)^{\otimes(p-1)} .
$$

To fix notation we assume that $\eta_{1}$ corresponds the left factor of the tensorproduct St $\otimes$ St in (31). Finally when applying the product map on each tensorfactor on the right hand side of (31) we obtain a map

$$
\mathrm{St} \otimes \mathrm{St} \rightarrow \mathrm{H}^{0}(2(p-1) \rho) \otimes \mathrm{H}^{0}(2(p-1) \rho) .
$$

Now $\eta$ is defined from (32) by applying the identification (23) . 
8.3. Maximal multiplicity. By the next result and Lemma 3.5 any element in the image of $\eta$ will be compatible with the diagonal.

Lemma 8.3. Every element within the image of (32) will vanish with multiplicity at least $(p-1) \operatorname{dim}(G / B)=(p-1)\left(\begin{array}{c}n \\ 2\end{array}\right)$ along the diagonal in $G / B \times G / B$.

Proof. Notice that by Lemma 7.1 every element within the image of $\eta_{\left\lfloor\frac{n}{2}\right\rfloor,\left\lceil\frac{n}{2}\right\rceil}$ will vanish with multiplicity at least $\left\lfloor\frac{n}{2}\right\rfloor$ along the diagonal. Thus by Lemma 8.1 it suffices to notice that

$$
\frac{n}{2}+2 \frac{n^{2}-2 n}{4}=\left(\begin{array}{l}
n \\
2
\end{array}\right)
$$

and

$$
\frac{n-1}{2}+2 \frac{(n-1)^{2}}{4}=\left(\begin{array}{l}
n \\
2
\end{array}\right)
$$

As a consequence, we may now consider $\eta$ as a map

$$
\eta: \mathrm{St} \otimes \mathrm{St} \rightarrow \operatorname{End}_{F}(G / B \times G / B, \operatorname{diag}(G / B))^{(1)} .
$$

8.4. A residual normal crossing. The only thing left is to prove that $\eta$ contains a Frobenius splitting in its image. For this, we start by observing

Lemma 8.4. Let $v_{-}$denote a lowest weight vector in St. Then the restriction of the function $\eta_{1}\left(v_{-}\right)$to $\left\{I_{n}\right\} \times U^{-}$coincides with $\mathfrak{f}_{n-1}^{p-1}$ up a nonzero constant. Similarly, the restriction of $\eta_{2}\left(v_{-}\right)$to $\left\{I_{n}\right\} \times U^{-}$ coincides with $\mathfrak{g}_{n-1}^{p-1}$ up to a nonzero constant.

Proof. Notice, first of all, that the map (27) will map $v_{-}$into the tensorproduct of the lowest weight spaces of the various $\mathrm{H}^{0}\left(\omega_{s}\right)$. Thus by Lemma 6.1 and the construction of $\eta_{1}$, the restricition of $\eta_{1}\left(v_{-}\right)$to $\left\{I_{n}\right\} \times U^{-}$will coincide with the product of the $(p-1)$-th powers of

$$
v_{\left(\left\lfloor\frac{s}{2}\right\rfloor+1, \ldots, s\right)},
$$

for $s=1,2, \ldots, n-1$. The latter product is by definition equal to $\mathfrak{f}_{n-1}^{p-1}$.

To obtain the statement about $\eta_{2}\left(v_{-}\right)$one first recalls that $\eta_{i, j}$, for $i+j>n$, was defined as the $\sigma$-twist of $\eta_{n-i, n-j}$. Hence, by Lemma 6.2, the element $\eta_{2}\left(v_{-}\right)$must equal the composition $\eta_{1}\left(v_{-}\right) \circ \tau$ up to a nonzero constant. The statement thus follows from the first part of the proof above.

Proposition 8.5. Let $\mathfrak{f}$ denote the image of $v_{-} \otimes v_{-}$under (32). Then the restriction of $\mathfrak{f}$ to $\left\{I_{n}\right\} \times U^{-}$coincides with the product

$$
\left(\mathfrak{f}_{n} \mathfrak{g}_{n-1}\right)^{p-1}
$$

up to a nonzero constant. 
Proof. By Lemma 8.4 we only have use Lemma 6.1 to observe that the restriction of the image of

$$
\eta_{\left\lfloor\frac{n}{2}\right\rfloor,\left\lceil\frac{n}{2}\right\rceil}: k \rightarrow \mathrm{H}^{0}\left(\omega_{\left\lfloor\frac{n}{2}\right\rfloor}\right) \otimes \mathrm{H}^{0}\left(\omega_{\left\lceil\frac{n}{2}\right\rceil}\right),
$$

to $\left\{I_{n}\right\} \times U^{-}$is generated by $v_{\left(\left\lfloor\frac{n}{2}\right\rfloor+1, \ldots, n\right)}$.

8.5. Frobenius splitting. We may now prove

Theorem 8.6. The G-equivariant map $\eta$ defines a canonical Frobenius splitting of $G / B \times G / B$.

Proof. Consider the commutative diagram

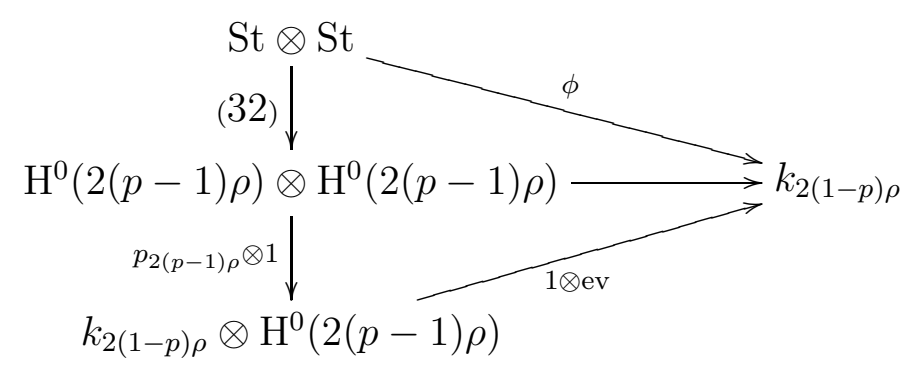

induced by the projection $p_{2(p-1) \rho}$ onto the lowest weight space and the $G$-equivariant evaluation map

$$
\text { ev }: \mathrm{H}^{0}(2(p-1) \rho)=\operatorname{End}_{F}(G / B)^{(1)} \rightarrow k .
$$

We claim that $\phi\left(v_{-} \otimes v_{-}\right)$is nonzero. To see this let $1 \otimes s$ denote the image of $v_{-} \otimes v_{-}$under the composed vertical map in (33). We have to prove that ev $(s)$ is nonzero. This can be checked locally on $U^{-}$ (considered as an open subset of $G / B$ ) where it follows by Proposition 8.5. Proposition 5.3 and the discussion in Section 4.1.

By construction $\phi$ is the composition of the $G$-equivariant map

(34) $\mathrm{St} \otimes \mathrm{St} \stackrel{\sqrt[32]{\longrightarrow}}{\longrightarrow} \mathrm{H}^{0}(2(p-1) \rho) \otimes \mathrm{H}^{0}(2(p-1) \rho) \stackrel{1 \otimes \mathrm{ev}}{\longrightarrow} \mathrm{H}^{0}(2(p-1) \rho)$,

with the projection map $p_{2(p-1) \rho}$. Moreover, by Frobenius reciprocity the composed map (34) is determined up to a constant. In particular, if $\phi$ is nonzero then (34) must coincide with the multiplication map

$$
\mathrm{H}^{0}((p-1) \rho) \otimes \mathrm{H}^{0}((p-1) \rho) \rightarrow \mathrm{H}^{0}(2(p-1) \rho),
$$

up to a nonzero constant. As a consequence (34) is surjective and thus it contains a Frobenius splitting of $G / B$ in its image. Hence (32), and thus also $\eta$, must contain a Frobenius splitting in its image.

Remark 8.7. The canonical Frobenius splitting $\eta$ is related to the concrete Frobenius splitting of $G / B \times G / B$ described in Section 4 of [6]. More precisely, in [Sect.4, loc.cit] one defines for $g, h \in G$ a new matrix $M(g, h)$ of size $2 n \times 2 n$. Next one considers the principal minors $\delta_{i}(M(g, h))$ of size $i$ (from the lower left hand corner) which defines sections of

$$
\mathrm{H}^{0}\left(\omega_{\left\lceil\frac{i}{2}\right\rceil}\right) \otimes \mathrm{H}^{0}\left(\omega_{\left\lfloor\frac{i}{2}\right\rfloor}\right)
$$


The concrete Frobenius splitting of $G / B \times G / B$ considered in [loc.cit] is then defined as the $(p-1)$-th power of the product

$$
\prod_{i=1}^{2 n-1} \delta_{i}(M(g, h))
$$

One may check that the elements $\delta_{i}(M(g, h))$ are connected to the above setup in the following way: when $1 \leq i \leq n$ the element $\delta_{i}(M(g, h))$ generates the image of the highest weight space under the map

$$
\eta_{\left\lceil\frac{i}{2}\right\rceil,\left\lfloor\frac{i}{2}\right\rfloor}: \mathrm{H}^{0}\left(\omega_{i}\right) \rightarrow \mathrm{H}^{0}\left(\omega_{\left\lceil\frac{i}{2}\right\rceil}\right) \otimes \mathrm{H}^{0}\left(\omega_{\left\lfloor\frac{i}{2}\right\rfloor}\right) .
$$

When $n<i \leq 2 n-1$ the element $\delta_{i}(M(g, h))$ generates the image of the lowest weight space under the map

$$
\eta_{\left\lceil\frac{i}{2}\right\rceil,\left\lfloor\frac{i}{2}\right\rfloor}: \mathrm{H}^{0}\left(\omega_{i-n}\right) \rightarrow \mathrm{H}^{0}\left(\omega_{\left\lceil\frac{i}{2}\right\rceil}\right) \otimes \mathrm{H}^{0}\left(\omega_{\left\lfloor\frac{i}{2}\right\rfloor}\right) .
$$

As a consequence, the concrete splitting in [loc.cit] coincide, up to a flipping of the factors of $G / B \times G / B$, with $\eta\left(v_{+} \otimes v_{-}\right)$for some highest weight vector $v^{+}$of St. In particular, $\eta\left(v_{+} \otimes v_{-}\right)$is compatible with with subvarieties of the form $X \times X$ for $X$ a Kempf variety in $G / B$.

\section{The SYMPLECTIC CASE}

In this section we will construct a canonical Frobenius splitting

$$
\bar{\eta}: \overline{\mathrm{St}} \otimes \overline{\mathrm{St}} \rightarrow \operatorname{End}_{F}(\bar{G} / \bar{B} \times \bar{G} / \bar{B}, \operatorname{diag}(\bar{G} / \bar{B}))^{(1)} .
$$

of $\bar{G} / \bar{B} \times \bar{G} / \bar{B}$ where any element in the image vanishes with multiplicity at least $(p-1) \operatorname{dim}(\bar{G} / \bar{B})$ along the diagonal. We start by constructing a certain residual normal crossing on $\bar{U}^{-}$.

9.1. The maps $\bar{\eta}_{i, j}$. Notice that the restrictions $\bar{\omega}_{i}$ and $\bar{\omega}_{n-i}$ of the fundamental characters $\omega_{i}$ and $\omega_{n-i}$ to $\bar{T}$ coincide. It follows that the $G$-equivariant maps $\eta_{i, j}$ in (12) induces similar maps in the symplectic case. E.g when $1 \leq i, j \leq 2 m$ and $i+j \leq 2 m$ we obtain, by restriction, an $\bar{G}$-equivariant morphism

$$
\bar{\eta}_{i, j}: \mathrm{H}^{0}\left(\omega_{i+j}\right) \rightarrow \mathrm{H}^{0}\left(\bar{\omega}_{i}\right) \otimes \mathrm{H}^{0}\left(\bar{\omega}_{j}\right)
$$

Elements in the image of $\bar{\eta}_{i, j}$ may be considered as functions on $\bar{G} \times$ $\bar{G}$. Actually these functions are, by definition, restrictions of certain functions on $G \times G$ which were described in Section 7 , In particular, by applying Lemma 7.1 and Lemma 7.2 , we may obtain a lower bound on the vanishing multiplicity along the diagonal in $\bar{G} \times \bar{G}$; e.g. with the bound $i+j \leq 2 m$ the multiplicity is at least $\min (i, j)$. 
9.2. Vanishing multiplicities. As in the case of $\eta$ the map $\bar{\eta}$ will be constructed using certain tensorproducts of the $\bar{\eta}_{i, j}$. In this case we consider the product of the maps

$$
\bar{\eta}_{\left\lfloor\frac{s}{2}\right\rfloor,\left\lceil\frac{s}{2}\right\rceil}: \mathrm{H}^{0}\left(\omega_{s}\right) \rightarrow \mathrm{H}^{0}\left(\bar{\omega}_{\left\lfloor\frac{s}{2}\right\rfloor}\right) \otimes \mathrm{H}^{0}\left(\bar{\omega}_{\left\lceil\frac{s}{2}\right\rceil}\right),
$$

for $s=1,2, \ldots, 2 m$, and

$$
\bar{\eta}_{m, 0}: \mathrm{H}^{0}\left(\omega_{m}\right) \rightarrow \mathrm{H}^{0}\left(\bar{\omega}_{m}\right) \otimes k .
$$

This leads to an $\bar{G}$-equivariant morphism (remember $\omega_{2 m}$ is the trivial character)

$$
\mathrm{H}^{0}\left(\omega_{m}\right) \otimes \bigotimes_{s=1}^{2 m-1} \mathrm{H}^{0}\left(\omega_{s}\right) \rightarrow \bigotimes_{s=1}^{m} \mathrm{H}^{0}\left(\bar{\omega}_{s}\right)^{\otimes 2} \otimes \bigotimes_{s=1}^{m} \mathrm{H}^{0}\left(\bar{\omega}_{s}\right)^{\otimes 2}
$$

The vanishing multiplicity along the diagonal in $\bar{G} \times \bar{G}$ for elements in the image of (35), is then described by the following result.

Lemma 9.1. Every element in the image of (35) vanishes with multiplicity at least $m^{2}=\operatorname{dim}(\bar{G} / \bar{B})$ along the diagonal in $\bar{G} \times \bar{G}$.

Proof. Applying Lemma 7.1 it suffices to observe the formula

$$
\sum_{s=1}^{2 m}\left\lfloor\frac{s}{2}\right\rfloor=m^{2}
$$

This ends the proof.

9.3. The construction of $\bar{\eta}$. We now want to construct the canonical Frobenius splitting $\bar{\eta}$. For this we first need to combine (35) with the following lemma.

Lemma 9.2. There exist nonzero $\bar{G}$-equivariant maps

$$
\overline{\mathrm{St}} \rightarrow \bigotimes_{s=1}^{m} \mathrm{H}^{0}\left(\omega_{s}\right)^{\otimes(p-1)}
$$

and

$$
\overline{\mathrm{St}} \rightarrow \bigotimes_{s=m}^{2 m-1} \mathrm{H}^{0}\left(\omega_{s}\right)^{\otimes(p-1)}
$$

both of them uniquely defined up to nonzero constants.

Proof. By dualizing the picture and using the selfduality of $\overline{\text { St }}$ we, first of all, have to show the existence and uniqueness, up to constants, of a nonzero $\bar{G}$-equivariant map

$$
\bigotimes_{s=1}^{m} \mathrm{H}^{0}\left(\omega_{n-s}\right)^{\otimes(p-1)} \rightarrow \overline{\mathrm{St}}=\operatorname{Ind} \frac{\bar{G}}{B}\left(-\sum_{s=1}^{m}(p-1) \bar{\omega}_{i}\right) .
$$

As $\overline{\emptyset_{i}}=\overline{\varnothing_{n-i}}$, for $i=1,2, \ldots, n-1$, this follows from Frobenius reciprocity and the remarks in Section 2.8. The second part of the statement follows in the same way. 
Lemma 9.2 enables us to compose the $(p-1)$-th tensorpower of (35) with the two morphisms in Lemma 9.2 . This way we obtain an $\bar{G}$ equivariant morphism

$$
\overline{\mathrm{St}} \otimes \overline{\mathrm{St}} \rightarrow \bigotimes_{s=1}^{m} \mathrm{H}^{0}\left(\bar{\omega}_{s}\right)^{\otimes 2(p-1)} \otimes \bigotimes_{s=1}^{m} \mathrm{H}^{0}\left(\bar{\omega}_{s}\right)^{\otimes 2(p-1)} .
$$

Actually as the left hand side of (35) contains two copies of the module $\mathrm{H}^{0}\left(\omega_{m}\right)$ there is some ambiguity about the definition of (39). There are several natural ways to construct (39) and all of them work equally well in the following. However, to be precise we fix the setup in such a way that the map (37) is associated to the copies of $\mathrm{H}^{0}\left(\omega_{m}\right)$ coming from the map $\bar{\eta}_{m, 0}$.

Composing (39) with the product morphism on each tensorfactor we next obtain the $\bar{G}$-equivariant map

$$
\overline{\mathrm{St}} \otimes \overline{\mathrm{St}} \rightarrow \mathrm{H}^{0}\left(\sum_{s=1}^{m} 2(p-1) \bar{\omega}_{s}\right) \otimes \mathrm{H}^{0}\left(\sum_{s=1}^{m} 2(p-1) \bar{\omega}_{s}\right),
$$

which by the relation

$$
\mathrm{H}^{0}\left(\sum_{s=1}^{m} 2(p-1) \bar{\omega}_{s}\right) \simeq \operatorname{End}_{F}(\bar{G} / \bar{B})^{(1)}
$$

then defines the $\bar{G}$-equivariant map of primary interest

$$
\bar{\eta}: \overline{\mathrm{St}} \otimes \overline{\mathrm{St}} \rightarrow \operatorname{End}_{F}(\bar{G} / \bar{B} \times \bar{G} / \bar{B})^{(1)} .
$$

9.4. A residual normal crossing. Observe that an element $g=\left(g_{i, j}\right)$ in $\bar{U}^{-}$is uniquely determined by the entries $g_{i, j}$ for $1 \leq j<i \leq 2 m$ and $i+j \leq n+1$. This follows directly from the relation (4) satisfied by elements in $\bar{G}$. In particular, we may identify $\bar{U}^{-}$with affine $m^{2}$-space $\mathbb{A}^{m^{2}}$ through the map

$$
\begin{gathered}
\bar{U}^{-} \rightarrow \mathbb{A}^{m^{2}}, \\
g \mapsto\left(g_{i, j}\right)_{\substack{1 \leq j<i \leq 2 m \\
i+j \leq n+1}},
\end{gathered}
$$

where we, for convenience, have indexed the coordinates in $\mathbb{A}^{m^{2}}$ by the set of pairs $(i, j)$ satisfying $1 \leq j<i \leq 2 m$ and $i+j \leq n+1$. The coordinate ring of $\mathbb{A}^{m^{2}}$ is then identified with $k\left[z_{i, j}\right]$ accordingly. Using the isomorphism (41) we also consider the coordinate ring $\bar{U}^{-}$as $k\left[z_{i, j}\right]$.

Recall that we in Section 5 introduced a collection $\mathfrak{f}_{r}, 1 \leq r \leq n$, of polynomial functions on $U^{-}$. In the following $\overline{\mathfrak{f}}_{r}, 1 \leq r \leq n$, will denote their restriction to $\bar{U}^{-}$.

Proposition 9.3. The polynomial $\overline{\mathfrak{f}}_{r} \in k\left[z_{i, j}\right]$ is a residual normal crossing relative to the set of variables

$$
\left\{z_{i, j}: 1 \leq j<i \leq n, i+j \leq r+1\right\},
$$


which are ordered using the order: $z_{i, j} \leq z_{i^{\prime}, j^{\prime}}$ if either $i+j<i^{\prime}+j^{\prime}$ or $i+j=i^{\prime}+j^{\prime}$ and $i \leq i^{\prime}$.

Proof. Recall the notation $k\left[Z_{i, j}\right], 1 \leq j<i \leq n$, for the coordinate ring of $U^{-}$in Section 5. On the level of coordinate rings the inclusion $\bar{U}^{-} \subset U^{-}$then takes the form

$$
k\left[Z_{i, j}\right] \rightarrow k\left[z_{i, j}\right]
$$

where

$$
Z_{i, j} \mapsto \begin{cases}z_{i, j} & \text { if } i+j \leq n+1, \\ f_{i, j} & \text { else, }\end{cases}
$$

for certain elements $f_{i, j}$ in $k\left[z_{i, j}\right]$. Now we may apply Lemma 5.1 and Lemma 4.3 to end the proof.

9.5. Frobenius splitting. Let $\bar{v}_{-}$denote a lowest weight vector of $\overline{\mathrm{St}}$. Before proving the main result we note the following result

Lemma 9.4. Let $\overline{\mathfrak{f}}$ denote the image of $\bar{v}_{-} \otimes \bar{v}_{-}$under the map (40). Then the restriction of $\overline{\mathfrak{f}}$ to $\left\{I_{n}\right\} \times \bar{U}^{-}$coincide with $\left(\overline{\mathfrak{f}}_{n}\right)^{p-1}$.

Proof. Notice, first of all, that the maps (36) and (37) will map $\bar{v}_{-}$into the tensorproduct of the lowest weight spaces of the various $\mathrm{H}^{0}\left(\omega_{s}\right)$. Thus by Lemma 6.1 and the construction of (40), the restriction of $\overline{\mathfrak{f}}$ to $\left\{I_{n}\right\} \times \bar{U}^{-}$will coincide with the product of the $(p-1)$-th powers of

$$
\bar{v}_{\left(\left\lfloor\frac{s}{2}\right\rfloor+1, \ldots, s\right)}
$$

for $s=1,2, \ldots, 2 m$. Here $\bar{v}_{\left(\left\lfloor\frac{s}{2}\right\rfloor+1, \ldots, s\right)}$ denotes the restriction of $v_{\left(\left\lfloor\frac{s}{2}\right\rfloor+1, \ldots, s\right)}$ to $\bar{U}^{-}$. The latter product is by definition equal to $\left(\overline{\mathfrak{f}}_{n}\right)^{p-1}$.

Theorem 9.5. The map $\bar{\eta}$ defines a canonical Frobenius splitting of $\bar{G} / \bar{B} \times \bar{G} / \bar{B}$. Moreover, every element in the image of $\bar{\eta}$ vanishes with multiplicty $(p-1) \operatorname{dim}(\bar{G} / \bar{B})$ along the diagonal. In particular, we may consider $\bar{\eta}$ as a map

$$
\bar{\eta}: \overline{\mathrm{St}} \otimes \overline{\mathrm{St}} \rightarrow \operatorname{End}_{F}(\bar{G} / \bar{B} \times \bar{G} / \bar{B}, \operatorname{diag}(\bar{G} / \bar{B}))^{(1)},
$$

where any Frobenius splitting in the image vanishes with maximal multiplicity along the diagonal.

Proof. By Lemma 9.1 we only have to prove that $\bar{\eta}$ contains a Frobenius splitting in its image. For this we proceed as in the proof of Theorem 8.6. Start by fixing a lowest weight vector $\bar{v}_{-}$in $\overline{\mathrm{St}}$ and consider the 
diagram ( $\mu$ denoting $\left.\sum_{s=1}^{m} 2(p-1) \bar{\omega}_{s}\right)$

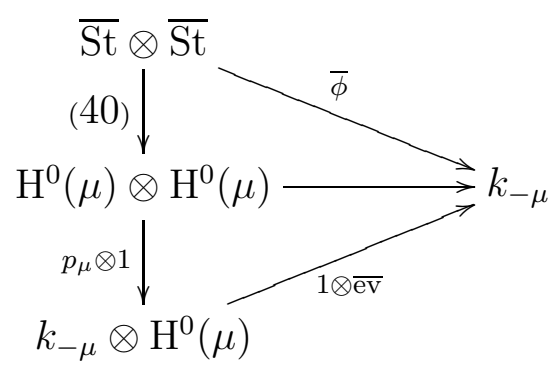

induced by the projection $p_{\mu}$ onto the lowest weight space and the $\bar{G}$-equivariant evaluation map

$$
\overline{\mathrm{eV}}: \mathrm{H}^{0}(\mu)=\operatorname{End}_{F}(\bar{G} / \bar{B})^{(1)} \rightarrow k .
$$

As in the proof of Theorem 8.6 it suffices to prove that $\bar{\phi}\left(\bar{v}_{-} \otimes \bar{v}_{-}\right)$is nonzero. To prove this let $1 \otimes \bar{s}$ denote the image of $\bar{v}_{-} \otimes \bar{v}_{-}$under the composed vertical map in (44). We have to prove that $\overline{\operatorname{ev}}(\bar{s})$ is nonzero. This can be checked locally on $\bar{U}^{-}$(considered as an open subset of $\bar{G} / \bar{B}$ ) where it follows by Lemma 9.4, Proposition 9.3 and the discussion in Section 4.1 .

\section{REFERENCES}

[1] Michel Brion and Shrawan Kumar, Frobenius splitting methods in geometry and representation theory, Progress in Mathematics, vol. 231, Birkhäuser Boston Inc., Boston, MA, 2005.

[2] Justin Brown and Venkatramani Lakshmibai, Wahl's conjecture for minuscule $G / P,(2008)$, arXiv:0809.2086v1 [math.AG] .

[3] Shrawan Kumar, Proof of Wahl's conjecture on surjectivity of the Gaussian map for flag varieties, Amer. J. Math. 114 (1992), no. 6, 1201-1220.

[4] V. Lakshmibai, V. B. Mehta, and A. J. Parameswaran, Frobenius splittings and blow-ups, J. Algebra 208 (1998), no. 1, 101-128.

[5] V. Lakshmibai, K. N. Raghavan, and P. Sankaran, Wahl's conjecture holds in odd characteristics for symplectic and orthogonal Grassmannians, Cent. Eur. J. Math. 7 (2009), no. 2, 214-223.

[6] N. Lauritzen, J. F. Thomsen Maximal compatible splitting and diagonals of Kempf varieties, (2010), arXiv:1004.2847v2 [math.AG].

[7] V. B. Mehta and A. J. Parameswaran, On Wahl's conjecture for the Grassmannians in positive characteristic, Internat. J. Math. 8 (1997), no. 4, 495-498.

[8] V. B. Mehta and A. Ramanathan, Frobenius splitting and cohomology vanishing for Schubert varieties, Ann. of Math. (2) 122 (1985), no. 1, $27-40$.

[9] Jonathan Wahl, Gaussian maps and tensor products of irreducible representations, Manuscripta Math. 73 (1991), no. 3, 229-259.

Institut for matematiske fag, Aarhus Universitet, 8000 Århus C, DENMARK

E-mail address: funch@imf.au.dk 